\title{
Meta-Review of Metanalytic Studies with Repetitive Transcranial Magnetic Stimulation (rTMS) for the Treatment of Major Depression
}

\author{
Bernardo Dell'Osso*, Giulia Camuri, Filippo Castellano, Vittoria Vecchi, Matteo Benedetti, \\ Sara Bortolussi and A. Carlo Altamura
}

\author{
Department of Psychiatry, University of Milan, Fondazione IRCCS Ca' Granda, Ospedale Maggiore Policlinico, \\ Milano, Italy
}

\begin{abstract}
Background: Major Depression (MD) and treatment-resistant depression (TRD) are worldwide leading causes of disability and therapeutic strategies for these impairing and prevalent conditions include pharmacological augmentation strategies and brain stimulation techniques. In this perspective, repetitive transcranial magnetic stimulation (rTMS) is a non-invasive brain stimulation technique with a favorable profile of tolerability which, despite being recently approved by the Food and Drug Administration (FDA) for the treatment of patients with medication-refractory unipolar depression, still raises some doubts about most effective parameters of stimulation. Methods: A literature search was performed using PubMed for the years 2001 through February 2011 in order to review meta-analytic studies assessing efficacy and safety issues for rTMS in depressive disorders. Fifteen meta-analyses were identified and critically discussed in order to provide an updated and comprehensive overview of the topic with specific emphasis on potentially optimal parameters of stimulation. Results: First meta-analyses on the efficacy of rTMS for the treatment of MD and TRD have shown mixed results. On the other hand, more recent meta-analytic studies seem to support the antidepressant efficacy of the technique to a greater extent, also in light of longer periods of stimulation (e.g. $>2$ weeks). Conclusion: rTMS seems to be an effective and safe brain stimulation technique for the treatment of medication refractory depression. Nevertheless, further studies are needed to better define specific stimulation-related issues, such as duration of treatment as well as durability of effects and predictors of response.
\end{abstract}

Keywords: Transcranial magnetic stimulation (rTMS), major depression (MD), treatment-resistant depression (TRD), meta-analyses.

\section{INTRODUCTION}

\section{Treatment Resistant Depression (TRD) and Therapeutic Options}

Major Depression (MD) is a severe and disabling condition with high levels of morbidity and mortality. Unfortunately, only about $50 \%$ of patients affected by MD respond to an initial course of antidepressants. For nonresponder subjects, strategies for achieving response include antidepressant switches, combination or augmentative therapies with different class of antidepressants, mood stabilizers, atypical antipsychotics and/or other psychotropic compounds. These options are not always well accepted by patients because of side effects, like weight gain and sexual dysfunctions, particularly in the long-term treatment. It has been estimated that approximately $2 \%$ to $15 \%$ of all patients with MD are refractory to any pharmacological treatment according to the specific adopted criteria [1,2]. For instance, treatment-resistant depression (TRD) has been defined as the lack of response to two antidepressant trials, given in

*Address correspondence to this author at the Department of Psychiatry, University of Milan, Fondazione IRCCS Ca' Granda, Ospedale Maggiore Policlinico, Via Francesco Sforza 35, 20122 Milano, Italy;

Tel: 02-55035994; Fax: 02-50320310;

E-mail: bernardo.dellosso@policlinico.mi.it succession, at adequate doses and for an adequate time frame, in compliant subjects [3]. For these patients, different treatment strategies are available, including pharmacological and psychotherapic augumentations as well as brain stimulation techniques [4]. The latters include electroconvulsive therapy (ECT), transcranial magnetic stimulation (TMS), deep brain stimulation (DBS), transcranial direct current stimulation (tDCS) and vagus nerve stimulation (VNS) [5]. Recently, among these techniques - which have different rationale, level of invasiveness and profile of tolerability repetitive TMS (rTMS) was approved by the Food and Drug Administration for the treatment of "patients with medication-refractory unipolar depression who have failed one good, but no more than one, pharmacological trial".

TMS has been introduced as neurophysiological technique in 1985, when Anthony Barker and his team developed a compact machine that allowed non-invasive stimulation of the cerebral cortex. Since its introduction, TMS has been used for the evaluation of the motor system, for functional studies of different brain areas and for the physiological study of several neuropsychiatric illness. Actually, over the last years, an increasing number of centers have started offering TMS for the treatment of different diseases, Major Depression in particular, condition TMS has already approved for in some countries. As a matter of fact, TMS has been considered by many clinicians even safer and better 
tolerated than many other pharmacological options or brain stimulation interventions (such as ECT) [6]. Nevertheless, questions about the magnitude of its antidepressant effect and about predictors of response in patients with TRD still remain open [7]. For instance, it has been established that a lower number of prior failed antidepressant treatments is associated with a better clinical outcome in relation to rTMS [8].

\section{TMS: Background and Rationale}

TMS generates a brief and intense magnetic field created by a strong electric current circulating within a coil resting on the scalp [9]. The powerful magnetic fields act as a vector that passes unimpeded across the skull and then converts into an electrical field within the brain. This procedure of brain stimulation through the scalp is not invasive and does not cause any pain at the surface [10]. The induced electrical stimulus activates a mixture of neurons beneath the coil. The magnetic field falls off rapidly with distance from the coil, so it is usually assumed that the stimulus activates neural elements in the cortex or subcortical white matter [11]. The stimulus, according to the stimulation intensity, is able to activate cortical neurons at a depth of $1.5-3.0 \mathrm{~cm}$ beneath the scalp. When using intensities below $120 \%$ of motor threshold, the stimulation cannot induce direct activation at depth of more than $2 \mathrm{~cm} \mathrm{[6].} \mathrm{Neuronal} \mathrm{axons,} \mathrm{rather} \mathrm{than} \mathrm{their} \mathrm{cell}$ bodies, are the most likely target of the stimulus as they have the lowest threshold for activation to the brief electrical current induced by TMS. However, coils with deeper power of stimulation have been recently introduced [12]. Different neurons have distinct threshold to electrical stimulation; low stimulation intensities, therefore, activate a much more limited selection of neurons than higher intensities. Most of our knowledge about the action of TMS on the human cortex comes from studies of the primary motor cortex. Stimulation of this area evokes activity in the muscle on the opposite side of the body, which is easily measured using electrophysiological methods. TMS of the motor cortex can detect the resting motor threshold and shows that the size of the response depends on the level of activity in the cortex at the time the stimulus is given and on the orientation of the coil on the head. TMS can be applied one stimulus at a time (single-pulse TMS), in pairs of stimuli separated by a variable interval (paired-pulse TMS), or in trains which is the most common stimulation setting used in psychiatric clinical practice (rTMS) [8]. Single pulses of TMS produce complex but short responses. Repeated pulses can have more prolonged effects on the modulation of cortical excitability, decreasing or increasing it. Relatively short-term effects (of the order of seconds or few minutes) are probably due to changes in neuronal excitability caused by shifts in ionic balance around populations of active neurons. Longer-lasting effects last usually 30-60 minutes and appear to depend on synaptic changes among cortical neurons, also known as long-term depression (LTD) and long-term potentiation (LTP) of synaptic connection. There is good evidence that changes resulting from TMS influence natural behaviours and have therapeutic potential. The nature of the after-effects of TMS depends on the site of stimulation and on the frequency, number and intensity of stimulation pulses, as well as on the pattern of the applied pulses [11].
The frequency at which the magnetic field oscillates differs between studies. Although different studies have used various frequencies, two main types have been used: low $(\leq 1$ $\mathrm{Hz})$ and high frequency $(>1 \mathrm{~Hz})$. The two types of stimulations have opposite effects and left and right hemispheres are supposed to have contrasting roles in mood regulation [13]. In particular, patients with depression seem to have reduced activity in the left prefrontal cortex. Therefore, many rTMS studies have been designed to excite this area with highfrequency stimulation and to achieve a long-lasting effect by applying rTMS during several daily sessions. More recently, investigators have focused on the hypotesis that in depressed patients there is an imbalance in the activity of the frontal lobes, with hypofunction in the left frontal lobe caused by excessive inhibition from the right one. This led to the idea that an alternative treatment would be low-frequency (suppressive) rTMS of the right prefrontal cortex [11]. Patterned rTMS refers to repetitive application of short rTMS bursts at a high inner frequency separated by short pauses of no stimulation. To date, most used protocols of this kind are the different theta burst stimulations (TBS) in which short bursts of $50 \mathrm{~Hz}$ are repeated at a rate in the theta range $(5 \mathrm{~Hz})$ as a continuous (cTBS) or intermittent (iTBS) train. In order to comply with present safety guidelines, protocols of slow rTMS generally apply all pulses in a continuous train, whereas protocols of fast rTMS apply shorter periods of stimulation separated by periods of pause. Obviously, there is a large variety of combinations of such protocols. Recently, quadripulse stimulation (QPS) has been added to patterned rTMS procedures to induce long-term changes of cortical excitability.

\section{TMS in Clinical Practice}

Several clinical trials have confirmed the overall safety of rTMS [14]. Induction of seizures is the most acute adverse effect; however, considering the small number of cases of accidental seizures induced by rTMS reported to date, the risk is certainly very low. More often than seizures, vasodepressor syncope may occur during TMS testing and treatment, due to anxiety and psycho-physical discomfort. Mania and hypomania have been reported in few bipolar and unipolar patients treated with TMS [15]. More frequently, minor side effects such as discomfort during the stimulation, local pain, neck pain, transient hearing changes and headache after stimulation, may occur [6].

Taken as a whole, the efficacy and safety of rTMS led the FDA, in October 2008, to approve a specific rTMS device that "is indicated for the treatment of Major Depressive Disorder in adult patients who have failed to achieve satisfactory improvement from one prior antidepressant medication at or above the minimal effective dose and duration in the current episode" [16]. This approval represents an important step forward for the diffusion of rTMS in the US and worldwide and should encourage further research.

With respects to predictors of response to rTMS, subjects with psychotic depression, those with depressive episode of longer duration, elderly subjects (prefrontal atrophy increases the distance between the coil and the cortex) and subjects with high degree of treatment resistance may be less likely to respond to rTMS [17]. Preliminary evidence sug- 
gests that clinical response to rTMS might itself be a predictor of the success of future treatments [13].

Given the robust number of clinical trials conducted with rTMS over the last decade, several meta-analyses have been progressively performed on the efficacy and safety of the technique.

The present review article was aimed to asses the efficacy and safety of rTMS in MD and TRD, in particular, providing an updated and comprehensive overview in the field. Specific emphasis was given on the comparison between recent and earlier rTMS studies and meta-analyses in order to evaluate changes in the efficacy of the technique due to a better definition of stimulation parameters in recent years. In fact, 10-year experience with rTMS in the treatment of MD should have optimized the parameters of stimulation, resulting in larger clinical effects as documented in recent trials using novel parameters of stimulation, such as more sessions or different intensity of stimulation [18].

\section{MATERIALS AND METHODOLOGY}

\section{Study Selection}

Articles for inclusion were identified conducting a literature search in PubMed in the period between January 1980 and December 2010. Search criteria were: "transcranial magnetic stimulation", "TMS", "rTMS", “depression”, "major depression, "depressive disorders", "treatment resistant depression" and "dysthymic disorder". Only papers published in English language were taken into account. For the purpose of our study, we decided to focus our attention on meta-analyses and systematic reviews, in particular. All reviewed studies included meta-analysis and randomized clinical trials (RCTs) that compared rTMS given at any frequency and localization, and controlled with sham intervention, in patients of any age and gender with a diagnosis of MD according to the DSM-IV criteria or treatment resistant depression [19, 20]. Meta-analysis is a statistical technique for amalgamating, summarizing and reviewing previous quantitative research. By using meta-analysis, a wide variety of questions can be investigated, as long as a reasonable body of primary research studies exist. Selected parts of the reported results of primary studies are entered into a database and this "meta-data" is "meta-analyzed", in similar ways to working with other data descriptively and then inferentially to test certain hypotheses.

\section{RESULTS}

Fifteen meta-analyses were retrieved (from 2001 to 2010), reviewed and summarized (Table 1).

\section{APPENDICES}

Table I. Summary of Metanalytic Studies (Focused on Efficacy) with rTMS Conducted in Major Depression to Date

\begin{tabular}{|c|c|c|c|}
\hline Study & Methods & Stimulation Parameters & Conclusions and Limitations \\
\hline McNamara et al., 2001 & $\begin{array}{l}\text { Number of trials: } 5 \\
\text { Avarage number of subjects } \\
\text { per trial: } 20-68 \\
\text { Total number of patients: } 151\end{array}$ & $\begin{array}{c}\text { Site: } 3 \text { left DLPC, } 1 \text { right DLPC, } 1 \\
\text { bilateral stimulation } \\
\text { Frequency: } 1-20 \mathrm{~Hz}\end{array}$ & $\begin{array}{l}\text { rTMS had beneficial effects on depression. There } \\
\text { was insufficient evidence to evaluate effect of rTMS } \\
\text { on mania and schizophrenia. } \\
\text { The duration of the antidepressant effects could not } \\
\text { be established. } \\
\text { Small number of trials. } \\
\text { Difficulty in blinding. }\end{array}$ \\
\hline Holtzheimer et al., 2001 & Number of trials: 12 & $\begin{array}{l}\text { Site: left and right DLPC (and } \\
\text { sham) }\end{array}$ & $\begin{array}{l}\text { rTMS was statistically superior to sham stimulation } \\
\text { in the treatment of depression, showing a moderate } \\
\text { to large effect size. The clinical significance of these } \\
\text { results was modest. }\end{array}$ \\
\hline Kozel and George, 2002 & $\begin{array}{l}\text { Number of trials: } 12 \\
\text { Avarage number of subjects } \\
\text { per trial: } 6-35 \\
\text { Total number of patients: } 230\end{array}$ & $\begin{array}{c}\text { Duration: } 2 \text { studies of } 1 \text { week and } 10 \\
\text { of } 2 \text { weeks } \\
\text { Site: } 12 / 12 \text { left DLPFC } \\
\text { Frequency: } 0.3-20 \mathrm{~Hz} \\
\text { Intensity: } 80-110 \%\end{array}$ & $\begin{array}{l}\text { There was a statistically significant evidence that left } \\
\text { DLPFC rTMS stimulation was an acute antidepres- } \\
\text { sant treatment. } \\
\text { Limits: small number of subjects per trial, different } \\
\text { parameters of stimulation, heterogeneity of the sam- } \\
\text { ple. }\end{array}$ \\
\hline Martin et al., 2001 & $\begin{array}{l}\text { Number of trials: } 16 \\
\text { Average number of subjects } \\
\text { per trial: } 19\end{array}$ & $\begin{array}{l}\text { Duration: } 1 \text { or } 2 \text { weeks; } \\
\text { Site: Left and right DLPFC, right } \\
\text { DLPFC (and sham). }\end{array}$ & $\begin{array}{l}\text { Positive global effect (HDRS) in favor of high- } \\
\text { frequency, left sided rTMS vs sham after } 2 \text { weeks. } \\
\text { This positive effect disappeared when using the BDI } \\
\text { scale. Small sample size. No description of method } \\
\text { of concealment allocation used. Double-blind re- } \\
\text { ferred to patient outcome assessor. }\end{array}$ \\
\hline $\begin{array}{l}\text { Burt et al., } 2002 \text { (first } \\
\text { analysis) } \\
\text { Open and uncontrolled } \\
\text { TMS studies }\end{array}$ & $\begin{array}{l}\text { Number of trials: } 13 \\
\text { Average number of subjects } \\
\text { per trial: } 16 \\
\text { Total number of patients: } 208\end{array}$ & $\begin{array}{c}\text { Duration: } 1-3 \text { weeks; } \\
\text { Site: Vertex, left and right DLPFC, } \\
\text { RF TMS, Motor area, bilateral } \\
\text { DLPFC TMS; } \\
\text { Frequency: from } 0,033 \text { to } 20 \mathrm{HZ} \text {; } \\
\text { Intensity: } 80-130 \% \text { or } 1-2,5 \mathrm{~T} \text {. }\end{array}$ & $\begin{array}{l}9 \text { studies reported quantitative change in depression } \\
\text { scores (HRSD and MADRS) but their average reduc- } \\
\text { tion was only } 37 \% \text { and the degree of therapeutic } \\
\text { change was relatively modest. } \\
\text { It should not be assumed that all studies derived from } \\
\text { the same population with the same characteristics. }\end{array}$ \\
\hline $\begin{array}{l}\text { (second analysis) } \\
\text { Randomized, controlled } \\
\text { studies }\end{array}$ & $\begin{array}{l}\text { Number of trials: } 23 \\
\text { Average number of subjects } \\
\text { per trial: } 19 \\
\text { Total number of patients: } 432\end{array}$ & $\begin{array}{l}\text { Duration: } 1-2 \text { weeks; } \\
\text { Site: Vertex, (frontal, temporal and } \\
\text { parietal) left and right DLPFC (and } \\
\text { sham); } \\
\text { Frequency: from } 0,17 \text { to } 40 \mathrm{~Hz} \text {; } \\
\text { Intensity: } 80-110 \% \text { or } 0,015-1,9 \mathrm{~T}\end{array}$ & $\begin{array}{c}\text { Slow and fast rTMS seemed to have statistically } \\
\text { superior antidepressant effects compared to sham but } \\
\text { the magnitude of the effects was of doubtful clinical } \\
\text { significance. The analysis of variance comparing } \\
\text { slow and fast rTMS did not yield a significant be- } \\
\text { tween-class effect. }\end{array}$ \\
\hline
\end{tabular}


Table I. contd....

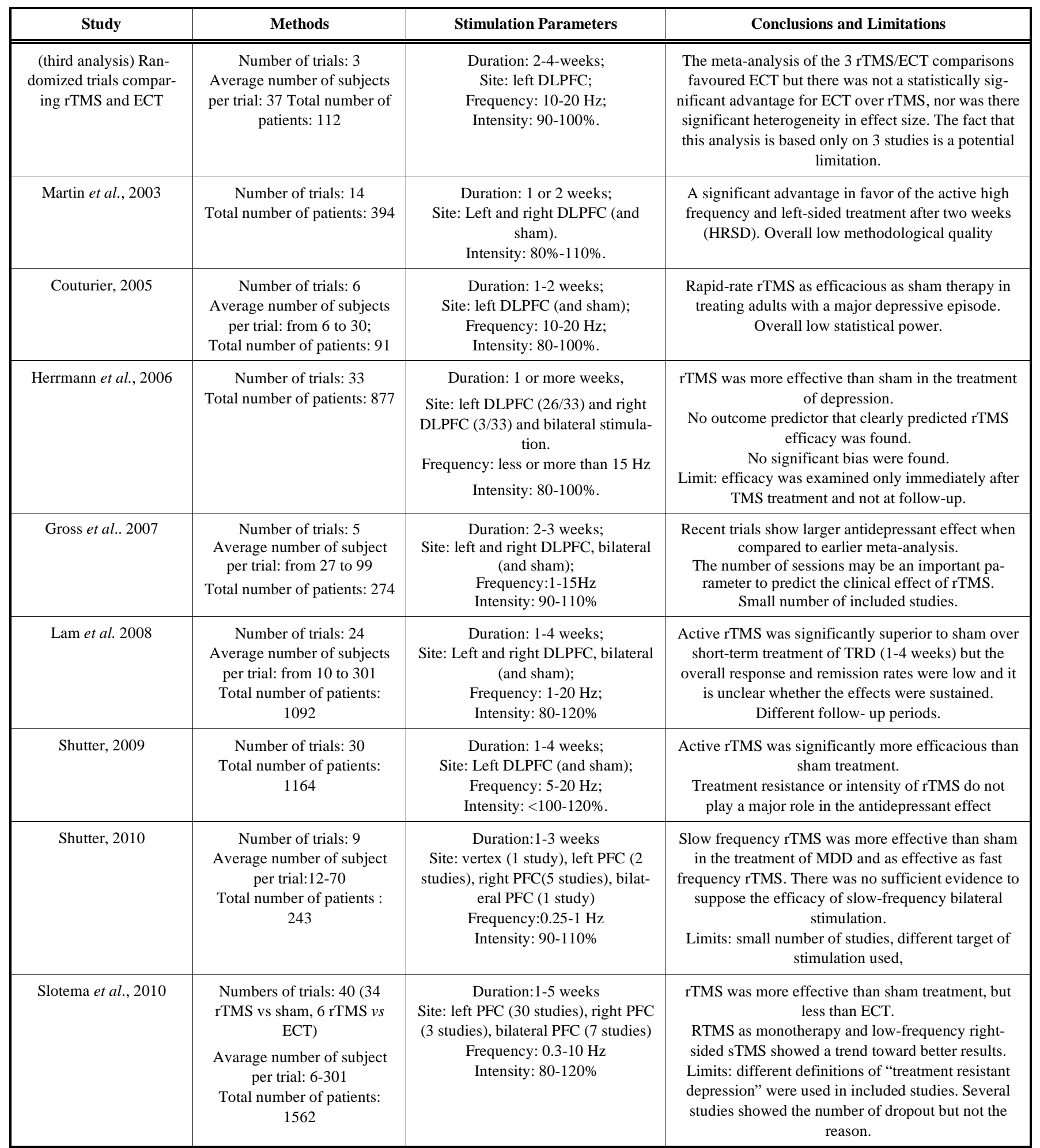

DLPFC: Dorso-Lateral-Pre-Frontal Cortex; Hz: Hertz; rTMS: repetitive Transcranial Magnetic Stimulation; HDRS: Hamilton Depression Rating Scale; BDI: Beck Depression Inventory; TRD: Treatment Resistant Depression

The first meta-analysis conducted by Martin and co-workers in 2001 [21] was aimed to assess the clinical efficacy and safety of the technique in MD. Sixteen RCTs were included and participants were patients with unipolar or bipolar depression in depressed phase. Fourty-five trials were excluded because of lack of or inadequate randomization, lack of a sham group, narrative review or descriptive studies with healthy volunteers. Different stimulation parameters were used. Of note, the mean duration of treatment was of 2 weeks. Several biases were found in the included 
studies so that the quality of this meta-analysis could not be optimal. In fact, some of these trials did not describe the randomization procedure making it difficult to blind the professionals who applied stimulation. For this reason, doubleblind design in these cases generally refers to the patient and to the outcome assessor (blind-rater design) and not to the clinician who performs the stimulation. Other limits were the small sample size of the studies (median $=19$ subjects), the use of different follow-up periods and the heterogeneity of the disorder. The Hamilton Rating Scale for Depression (HRSD) [22] was used as a primary outcome measure and authors found a positive global effect in the comparison between high-frequency, left sided TMS vs placebo after 2 weeks. However, this positive effect disappeared when using the Beck self-applied questionnaire (BDI) [23]. In addition, low frequency, right-sided rTMS appeared more effective than sham after 2 weeks of treatment (but not at one week). Finally, when rTMS was compared to ECT, significant differences appeared in favor of ECT only when patients had psychotic symptoms. In conclusion, this meta-analysis suggested that there was no strong evidence for a clear efficacy of TMS for the treatment of depression, even though the results did not exclude the possibility of benefit.

In 2001, another meta-analysis by McNamara and colleagues assessed the effectiveness of rTMS in mood disorders and schizophrenia [24]. Sixteen studies of rTMS in TRD were identified, but only 5 were analyzed being these conducted vs sham. In 3 studies rTMS was applied at 10-20 $\mathrm{Hz}$ to the left frontal region. In one study it was applied at 1 $\mathrm{Hz}$ to the right side and in the other trial both techniques were applied. Taken as a whole, these 5 trials enlisted 151 patients: 81 were treated and 70 were in the control group. The duration of treatment varied between 1-4 weeks (2 weeks of stimulation plus 2 weeks of sham). The overall chisquared test of association implied a statistically significant benefit of rTMS. The difference between rates of improvement in the treated and control groups was $43 \%$. In terms of tolerability and adverse events, no seizure was reported and the only side-effect was transient headache. The major limit consisted of the lack of a proper sham control. The duration of antidepressant effect after rTMS treatment, moreover, was not specifically defined in any of these studies and, finally, the small size of these trials represented another limitation. In conclusion, the authors reported a positive effect for TMS, but could not establish which stimulation parameters are the more effective and which patients are more likely to respond to the procedure.

In the same year, Holtzheimer and colleagues (2001) identified 12 RCTs of TMS in MD, 11 of which identifying the left dorsolateral pre-frontal cortex (DLPFC) as stimulation target. Fixed and random effects meta-analyses were performed on these studies comparing the decrease in HRSD scores obtained with rTMS and sham stimulation. However, given that initial results with a fixed-effects analysis failed homogeneity testing, a random-effects analysis was used to calculate all results. In 12 studies, the weighted mean effect size was 0.81 . For studies using left DLPFC stimulation, the weighted mean effect size was 0.89 and, for studies using left DLPFC stimulation in a parallel-groups design (7 studies), the weighted mean effect size was 0.88 . Of clinical interest, no study showed a mean decrease in HRSD scores >
$50 \%$; in fact, only few patients were considered HRSD responders after the overall stimulation $(13.7 \%$ with rTMS vs $7.9 \%$ with sham). Authors concluded that rTMS was statistically superior to sham stimulation in the treatment of depression, showing a moderate to large effect size. Nonetheless, authors remarked that the clinical significance of these results was modest [25].

The study conducted by Burt and co-workers in 2002 [26] included 3 distinct meta-analyses assessing effect size and magnitude of therapeutic effects of rTMS. The first meta-analysis was performed on open and uncontrolled trials of rTMS in the treatment of MD. Nine trials reported significant quantitative changes in depression scores and the weighted effect size confirmed the antidepressant effect of both slow or fast rTMS. Nevertheless, the average reduction in HRSD and Montgomery-Asberg Depression Rating Scale (MADRS) scores was around $37 \%$ and the clinical significance of this effect was uncertain. The second meta-analysis involved 23 sham or otherwise controlled studies with rTMS. Across these comparisons, the combined effect sizes indicated a moderate to large effect and there was only one comparison with a clearly negative effect size. Results indicated that slow and fast rTMS had statistically superior antidepressant properties compared to sham stimulation. The analysis of variance in relation to slow and fast rTMS showed that higher frequency stimulation did not necessarily enhance the antidepressant effect. Despite these positive results, the effect size was not so impressive and the mean improvement in HRSD scores between active and control condition was only $16 \%$. Finally, in a third meta-analysis, 3 trials which had randomly assigned depressed patients to treatment with rTMS or ECT were analyzed. Results indicated that changes in the HRSD scores favored ECT over rTMS, but this difference was not statistically significant.

In the same year, Kozel and George [27] selected 12 randomized sham-controlled trials of left prefrontal rTMS in MD in their meta-analysis, with a total sample of 230 patients. The sample resulted homogeneous and the cumulative effect size was 0.53. Days of treatment varied from 5 to 10 . The outcome variable for all included studies was defined as the change in HRSD score. The cumulative effect sizes were statistically significant and the improvement clinically measurable, leading the authors to support the hypothesis that left rTMS over the DLPFC is an efficacious instrument to treat depression, in particular for acute treatment. Principal limitations of this meta-analysis were the inclusion of studies with a small number of subjects and the heterogeneity of patients included. Moreover, data about duration of improvement and about the relationship between concomitant medication use and TMS effect were not reported. The authors concluded suggesting that the best parameters for treatment may depend on regional brain blood flow prior to treatment [28].

In 2003, Martin and co-authors conducted another study to assess the efficacy of rTMS in MD by performing a systematic review of RCTs comparing rTMS with sham stimulation [29]. Fourteen trials were included according to the following criteria: all were randomized trials with rTMS given at any frequency and at any localization with a sham intervention in patients of any age and gender with a diagno- 
sis of unipolar or bipolar depression, with or without psychotic symptoms according to DSM-IV or ICD-10 criteria. Forty-eight studies were excluded. Clinical heterogeneity in selected trials was due to differences in the target of stimulation, duration of treatment and number of interventions per day (one or more). The majority of these studies compared left-sided, high-frequency rTMS with a sham group; only one study compared right-sided, low-frequency TMS with sham. In 9 studies treatment duration was 2 weeks, while in the others just 1 week. All studies used the HRSD as primary outcome measure and 9 used the BDI as well. Quality of most studies was generally low and the majority of them did not provide a precise description of randomization process and none described the method of concealing. Only 2 studies undertook an intention-to-treat analysis. Most studies presented as double-blind trials were, actually, single-blind trials with blind-rater evaluations. Only 3 studies stated that patients had been free from antipsychotic medications for 1 week before entering the study [30-32]. Other limitations were the small sample size of the selected studies (median = 29 subjects) and the difficulty of interpreting results from psychometric scales [33]. In terms of results, a small effect was detected in favor of the active group when measured by HRSD after 2 weeks of high frequency, left-sided rTMS, but not at 2 weeks of follow-up.

In 2005, a systematic review and meta-analysis by Couturier [34] assessed the efficacy of rapid-rate rTMS in the treatment of MD on the basis of studies conducted from 1966 until 2003. Criteria pertaining to intervention were a) rapid rTMS, high frequency $(10 \mathrm{~Hz}), \mathrm{b})$ application over the left DLPFC, c) intensity (80\%), and d) the presence of sham control. These inclusion criteria were satisfied by 6 studies (3 of which were crossover trials) which had a duration of 1 or 2 weeks. The results indicated that rapid-rate rTMS was not more efficacious than sham therapy in treating depression. These results are different from those of a previous meta-analysis on 5 RCTs by McNamara and coworkers [24] who found a beneficial effect of rTMS compared with placebo, as shown before. In the Couturier' meta-analysis, 3 possible explanations for the lack of benefit for rTMS versus sham therapy were provided. The first one was simply that rTMS had a similar efficacy to sham. The second was the lack of definition of effective stimulation parameters of rTMS. In particular, Couturier suggested that at that time there was no evidence about the relevance of DLPFC as stimulation target. With respect to stimulation parameters of the trials included in the meta-analysis, particularly in relation to the duration of stimulation, it was maximum 2 weeks. Another confounding variable may have been the nature of sham condition, because inadequate sham procedure may partially stimulate the cortex, exerting possible therapeutic effects [35]. Finally, the author stressed the low statistical power of the 6 studies included in the analysis (too small samples despite being overall well-designed studies). Heterogeneity in these studies was not significant, indicating that data source was appropriate.

A meta-analysis conducted by Herrmann's group in 2006 was aimed to investigate optimal parameters in relation to rTMS response through a literature search of controlled trials [36]. Thirty three TMS studies conducted on depressed patients were included in the random-effect meta-analysis and secondary analyses comparing outcome of studies with different parameters were conducted as well. Principal inclusion criteria were: randomization in parallel or crossover design with sham, blinding of both patient and investigator, diagnosis of unipolar or bipolar depression (only in 1 study participants suffered from post-stroke depression) [37]. Studies had to report results by using the HRSD or the MARDS. Separate meta-analyses were conducted according to the classification of different studies in the following groups: mean age: $<50$ versus $\geq 50$ years; treatment resistance (yes/no); number of TMS sessions (5, 10, more than 10); type of depressive disorder; medications (stable or not); stimulation intensity $(80 \%-100 \%$ or more than $100 \%)$; left DLPFC stimulation frequency (in some studies frequency was less than $15 \mathrm{~Hz}$ and in others more than $15 \mathrm{~Hz}$ ); presence of psychotic features, etc... In order to assess the quality of each study, a specific rate according to Couturier's criteria was performed as well. Results showed that rTMS was more effective than sham (with a large effect size of 0.071 and a mean reduction of depressive symptoms of $33.6 \%$ ), without significant differences between studies previously included and excluded in Couturier's meta-analysis. No significant relationship between trials quality and effect size was found. Probably due to the high variability in stimulation parameters, variables that could clearly predict TMS response were not identified. Nevertheless, studies included patients on unstable medications and studies using intensity < $90 \%$ of the motor threshold may have resulted in lower level of efficacy. DLPFC high frequency and low frequency appeared to be equally effective [38].

Gross and colleagues in 2007 [39] conducted a systematic review and meta-analysis to specifically investigate whether more recent studies (published from December 2005 to November 2006) on rTMS in MD, using different parameters of stimulation, have shown improved clinical results compared to previous meta-analyses. Inclusion criteria were similar to those used in the Martin's meta-analysis and 5 studies were included. Heterogeneity among these 5 trials consisted of the site and frequency of stimulation (bilateral stimulation in one study, low frequency right-sided rTMS in two studies and high frequency left-sided in the others), number of sessions (from 10 to 16), use of different outcome measures (e.g., one study used the MADRS while the others the HRSD) and different levels of treatment resistance. The quality of the overall analysis was significantly improved. The number of patients was 274, mean age 44.7 years, and female to male ratio 183:91. Of note, authors found a larger antidepressant effect of TMS when compared with previous studies, both in low-frequency right-sided [40] and in highfrequency left-sided TMS [41, 42]. Of clinical interest, in relation to high-frequency, left-sided rTMS, there were significant differences between previous metanalytic studies and this meta-analysis, particularly when considering the number of sessions (10 vs 15). In fact, it was shown that the number of sessions might be an important parameter to predict the clinical effect of rTMS. The same conclusion was reported from studies stimulating in low frequency, right side $[43,44]$. Another potential difference in patient selection was that patients enrolled in more recent studies were 
less refractory to medications and, therefore, more likely to respond. The comparison of the effect size of 2 studies of bilateral stimulation suggested that bilateral stimulation did not induce a larger effect size $(0.59$ and 0.84 vs 0.82 and 1.49 for the 2 bilateral stimulation, unilateral left and unilateral right stimulation studies, respectively). The possibility to draw any significant conclusion was limited by the small number of studies.

The meta-analysis conducted by Lam and colleagues in 2008 [45] systematically reviewed published studies with rTMS in patients affected by TRD, focusing on clinical outcome. A total of 32 RCTs comparing rTMS with sham control were identified and 8 were excluded due to the lack of sham treatment [46-48], no definition of TRD [49,50], or because the major aim of the studies was to analyze improvement of cognitive function or reduction of pain in patients with MD [51,52], or multiple crossover with no data on the first treatment sequence [53]. One of the remaining studies did not report clinical response, remission rates and rating scales scores [54]. As a consequence, only 23 studies were available for quantitative analysis. The primary outcome measure was clinical response, defined as the reduction $\leq$ than $50 \%$ on HDRS or MADRS scores. Other outcome measures included clinical remission, defined as the presence of endpoint scale scores within the normal range and the standardized mean difference. Nine studies considered TRD as the failure of $\geq 1$ trial of antidepressants; the others as the failure of $\geq 2$. There was homogeneity of variables, with high frequency left-rTMS as the most common studied active condition. Most of the studies used 1 to 2 weeks of treatment; 3 RCTs used 3 weeks and only 2 studies 4 weeks. In the majority of the studies, patients were taking concomitant antidepressants, with dosages kept unchanged before and during rTMS. Only 4 studies included drug-free patients. In 2 studies, patients started a new antidepressant at the same time of TMS $[55,56]$. Only 8 studies systematically followed-up patients after completing the double-blind phase, usually in the short-term (1 to 3 weeks). Given that different studies reported that longer treatment period are associated with higher response rates, the principal limitation of this meta-analysis is the short term duration (1-2 weeks) of most studies. Results showed that antidepressant effects persisted for 1 to 2 weeks after TMS discontinuation. Two RCTs had longer follow-up periods: in the first one, rTMS was started together with $20 \mathrm{mg}$ of escitalopram showing maintenance of rTMS response for 5 weeks but no difference was found at 9 weeks [56]. The second did not show significant difference between active and sham at 5 weeks. Main results of this meta-analysis showed that active rTMS was significantly superior to sham treatment over short-term acute treatment of TRD (1-4 weeks). This meta-analysis also reported a good tolerability of rTMS, with few dropouts and side-effects. Despite this positive result, the overall response and remission rates were low and it was unclear whether rTMS effects were sustained.

In 2009, Shutter et al. [57] published a meta-analysis that included a group of studies with high frequency rTMS over the left DLPFC in MD published between January 1980 and November 2007. A total of 30 double-blind sham-controlled studies were included with 1164 patients enrolled, 606 of whom received real rTMS and 558 sham treatment. The overall weighted mean effect size for treatment was 0.39 showing that rTMS was significantly more effective than sham. Differences in the effect size between treatment resistant and non-resistant patients were not found. Finally, the comparison of effect sizes between studies that applied stimulation intensities $<100 \%$ MT $(n=14)$ and studies that used $100-120 \%$ intensities $(n=16)$ were not found, supporting the hypothesis that intensity of rTMS does not play a major role in term of antidepressant effect. The authors stressed a major methodological limitation in the revised studies which was the unavailability of an ideal sham condition; in fact, the procedure of sham stimulation used in many trials could have been partially active and the physician could not be really blind to it.

Recently Shutter [58] conducted a systematic research in order to examine the efficacy of slow-frequency rTMS to the frontal cortex in MD. Included articles were identified through a literature search in the period between January 1994 and July 2009. The author examined, in a randomeffect meta-analysis, 9 double-blind sham-controlled parallel intention-to-treat studies. The total sample included 252 patients with a mean age of 50 years. The mean number of treatment sessions in these studies was 9 (5 to 16 session) and the mean intensity of stimulation was $102 \%$. The first aim of the study was to assess the efficacy of slow-frequency rTMS by analyzing the effects of sham vs real stimulation in depressed patients. The cumulative effect size for treatment was 0.63 which supported the efficacy of TMS. The second aim was to compare slow and fast-frequency stimulation, and results of this random-effect model analysis did not show any significant difference between the two procedures. The major limitations of this study was the small number of double-blind sham-controlled studies which might have underpowered its results and the large variation of target stimulation sites. Moreover, the available data were not suitable to determine effect sizes for low-frequency rTMS applied over the left and right frontal prefrontal cortex separately. Author's conclusion was that slow-frequency was as effective as fast frequency, though generally better tolerated, allowing longer and safer stimulation periods.

Of note, Brunoni and colleagues [59] have recently (2009) published a meta-analysis in order to assess the magnitude of the placebo response in MD. A literature search was performed from April 2002 to April 2008 and forty-one studies were included; 29 studies used TMS and 12 escitalopram vs sham. Results showed a large placebo response for both TMS and escitalopram, suggesting that sham response is related with previous treatment refractoriness and with the use of TMS as add-on strategy, but not with other variables like age and gender

In 2010, Slotema et al. [60] published a meta-analysis that included studies with rTMS in MD ( $\mathrm{n}=40)$, auditory verbal hallucinations $(n=7)$, negative symptoms of Schizophrenia $(\mathrm{n}=7)$, Obsessive-Compulsive Disorder $(\mathrm{n}=3)$, Tourette's Syndrome $(n=2)$, Panic Disorder $(n=1)$, Bulimia Nervosa $(n=1)$, Mania $(n=1)$ and Post-Traumatic Stress Disorder $(\mathrm{n}=1)$. A literature search was performed from 1966 till 2008. With respect to rTMS in MD, studies 
were divided in 2 groups: rTMS vs sham (34) and rTMS vs ECT (6). With respect to the first group, a total of 1347 patients was enrolled, 715 of whom received real rTMS and 632 sham treatment. The mean weighted effect size for all studies was 0.55: for left-sided rTMS it was 0.53 , for rightsided rTMS 0.82 and, finally, for bilateral rTMS 0.47. TMS as a monotherapy (effect size $=0.96$ ) was compared to rTMS started simultaneously with medication $($ effect size $=0.51$ ) or during continuation of preexisting antidepressant treatment as augmentation treatment (effect size $=0.37$ ). With respect to the second group, a total of 215 patients was enrolled (113 treated with rTMS and 102 with ECT), showing greater efficacy for ECT, with a weighted effect size of 0.47 . Results indicated that TMS was more effective than sham in the treatment of MD, but less effective than ECT. Moreover, there was a trend for rTMS for being more effective as monotherapy. Authors' conclusion was that rTMS deserved a place in standard treatment for TRD and other psychiatric disorders.

To date and to authors' knowledge, 2 main meta-analyses have been specifically focused on the safety of rTMS. A meta-analysis conducted by Machii and colleagues in 2006 [61] was aimed to assess the safety of rTMS applied over non-motor areas. A specific search of any adverse effect was conducted considering all articles published from January 1998 to December 2003. Headache was the most common side effect, occurring in $23 \%$ of patients. Major side effects were rare and consisted of 2 seizures and 4 cases of psychotic symptoms induced by left-sided rTMS. In one case of seizure, rTMS was applied to the left DLPFC at an intensity of $120 \%$ and a frequency of $15 \mathrm{~Hz}$ [62]. In the other, rTMS was applied over the left DLPFC at an intensity of $110 \%$ and a frequency of $20 \mathrm{~Hz}$ [63]. Taken as a whole, the frequency adopted in these two cases is higher than what is generally used $(10 \mathrm{~Hz})$. In conclusion, rTMS of non-motor areas appeared to be safe with few and mild side-effects.

A recent meta-analysis conducted by Janicak and colleagues in 2008 [64] assessed the safety of rTMS given that prior studies had given only limited importance to this aspect. In addition, the acute efficacy of rTMS and the duration of its benefit were analyzed. Three separate clinical protocols were included, with 325 patients from 23 clinical sites in the US, Australia and Canada. All studies concerned the use of rTMS in the treatment of MD. Safety assessment was conducted in relation to side effects, cognitive functions and auditory threshold. TMS was administered in over 10000 cumulative treatment sessions in this study program. Most frequent side effects were mild, such as headaches and scalp discomfort. No adverse events such as seizures were reported. Auditory threshold and cognitive function did not change during the stimulation. Discontinuation rate due to adverse events was found to be around $4.5 \%$. In conclusion, rTMS was well tolerated and associated with low incidence of side effects that were mostly mild to moderate.

\section{DISCUSSION}

The results of retrieved meta-analyses rise different questions about the state of art of TMS in MD. On one hand, it is clear that first meta-analyses showed mixed results on the efficacy of rTMS for the treatment of TRD [24, 26, 27]. In particular, the attention was focused on high frequency stimulation, that resulted fairly better than placebo [21]. In the following years, some doubts persisted about the real efficacy of the technique and many authors confirmed that there was insufficient evidence to view rTMS as effective treatment for depression $[29,34]$ and to predict treatment response [36], whereas other authors supported the antidepressant effect of rTMS $[39,56]$, particularly in the acute treatment [45]. On the other hand, the most recent metaanalyses [58] seemed to support the efficacy of low frequency rTMS and confirmed that low and rapid-rate rTMS are equally effective in the treatment of TRD. RTMS questioned efficacy in first meta-analyses may be explained taking into account the following factors. First of all, the low statistical power of many meta-analyses, which is related to the small size of the analyzed samples. Nevertheless, as Gross and colleagues showed in 2007, evidence from studies using high-frequency, left-sided rTMS showed significant differences compared with previous studies and, in this perspective, the meta-analysis by Gross stressed the importance of the number of sessions ( $10 \mathrm{vs} 15$ sessions). In fact, it has been shown that the number of sessions is an important parameter to predict the clinical effect of rTMS. The same conclusion was reported when it was stimulated the right prefrontal cortex at low frequency, with results comparable to the high-frequency, left-sided stimulation. Therefore, the duration of treatment is supposed to play a very important role in the antidepressant efficacy of rTMS. This aspect has been further stressed in a review by Loo and colleagues [65] that showed that 2-week treatment course provided only modest clinical outcomes and was additionally remarked by Daskalakis and coworkers in 2008 [66]. In the same perspective, O'Reardon and colleagues clearly showed that a course of stimulation longer than 2 weeks of rTMS is required for the achievement of significant improvement, observing, moreover, that 2 additional weeks of rTMS beyond the initial 4 weeks could result in further clinical benefit [67].

Other reasons for the modest efficacy of rTMS compared to sham stimulation include the different definitions used for TRD, as well as the different adopted procedures of sham stimulation and the use of different clinical outcome measures. To join a general consensus about research and clinical settings, safety, ethical consideration and stimulation parameters, safety and efficacy guidelines on brain stimulation and, in particular on TMS, have been recently edited $[8,68]$. In fact, over the last years the number of applications of TMS has substantially increased, with the adjustment of previous paradigms and the development of novel ones (e.g., pattern repetitive TMS). Moreover, technical advances have led to new device design and to the integration of TMS with electroencefalography (EEG), positron emission tomography (PET) and functional magnetic resonance imaging (fMRI). Finally, treatment guidelines are necessary for safety reasons in order to establish appropriate and safe range of stimulation in terms of frequency, intensity, etc... In fact, even though the occurrence of seizures has been extremely infrequent, it is definitely important to avoid this adverse event stimulating within safe parameters and excluding potential 
risk factors [8]. On the other hand, if we can be optimistic about the efficacy of rTMS in the short-term treatment of $\mathrm{MD}$ and TRD, it is unclear whether these beneficial effects are sustained [36, 45, 69] given that follow-up studies are substantially lacking.

In this perspective, a recent study has investigated the long-term efficacy of rTMS in bipolar patients, suggesting that immediate remission is predictive of sustained benefit after 1 year [69]. Another recent study conducted by Janicack et al. [70] examined the persistence of rTMS benefits in a 24 weeks follow-up, finding that patients who benefited from acute treatment mainteined this effect in the long-term while on mainteinance antidepressant monotherapy.

\section{CONCLUSION}

Traditionally used in neurophysiology as a research tool, rTMS has been applied during the last decade in a variety of psychiatric disorders - mostly MD with treatment resistant features - as a potentially therapeutic intervention. Most of the work in this field has been carried out in drug-resistant patients with positive results emerging from recent metaanalyses which analyzed studies using novel and more effective stimulation parameters (e.g., a greater number of sessions). The multitude of parameters of stimulation implicated substantially different settings in many studies, but metanalytic indications help us to start to better define important practical aspects in rTMS treatment, such as the duration, stimulation target, frequency and definition of the degree of treatment resistance and of the predictors of response. Safety continues to be an important point favoring the clinical use of TMS, even though it is crucial that this intervention is provided by centers and clinicians with proven experience in the field. In terms of tolerability, it may be worthwhile to mention recent data from the Optimization of TMS for the Treatment of Depression (OPT-TMS) study, reporting a significant decrease of procedural pain over time of leftprefrontal rTMS for Depression. OPT-TMS is a 4 year, multicenter, double-blind, randomized, sham-controlled study with daily left prefrontal rTMS for MD, including 240 patients. Initial results from the open-label phase of this trial, in fact, suggest that painfulness of left prefrontal rTMS diminished of $48 \%$ over 3 weeks of daily treatment [71].

\section{DISCLOSURE}

The authors do not have any conflict of interest with the content of the present article.

\section{REFERENCES}

[1] Berlim MT, Turecki MG. Using psychostimulants for treating residual symptoms in major depression. J Psychiatry Neurosci 2007; 32(4): 304.

[2] Nemeroff CB. Prevalence and management of treatment-resistant depression. J Clin Psychiatry 2007; 68(Suppl 8): 17-25.

[3] Sackeim HA. The definition and meaning of treatment-resistant depression. J Clin Psychiatry 2001; 62(S16): 10-7.

[4] Demitrack MA, Thase ME. Clinical significance of Transcranial Magnetic Stimulation (TMS) in the treatment pharmacoresistant depression: Synthesis of Recent Data. Psychopharmacol Bull 2009; 42(2): 5-38.

[5] Dell'Osso B, Camuri G, Oldani L, Altamura AC. Current and future perspectives on vagus nerve stimulation in treatment-resistant depression. In: Rogers EMH, Anderson PB, Eds. Deep brain stimu- lation: applications, complications and side-effects. Nova Science Publisher: New York 2009; pp. 177-88.

[6] Rossi S, Hallet M, Rossini P, Pascual-Leone and The Safety of TMS Consensus Group. Safety, ethical considerations, and application guidelines for the use of transcranial magnetic stimulation in clinical practice and research. Clin Neurophysiol 2009; 120(12): 2008-39.

[7] Padberg F, George MS. Repetitive transcranial magnetic stimulation of the prefrontal cortex in depression. Exp Neurol 2009; 219(1): 2-13.

[8] Brakemeier EL, Luborzewski A, Danker-Hopfe H, Kathmann N, Bajbouj M. Positive predictors for antidepressive response to prefrontal repetitive transcranial magnetic stimulation (rTMS). J Psychiatr Res 2007; 41(5): 395-403.

[9] Rossini P, Rossi S. Trancranial magnetic stimulation: diagnostic, therapeutic, and research potential. Neurology 2007; 68(7): 484-8.

[10] Dell'Osso B, Mundo E, D'Urso N, et al. Augmentative repetitive navigated transcanial magnetic stimulaton (rTMS) in drug-resistant bipolar depression. Bipolar Disord 2009; 11(1): 76-81.

[11] Ridding M, Rothwell J. Is there a future for therapeutic use of transcranial magnetic stimulation? Nat Rev Neurosci 2007; 8(7): 559-67.

[12] Harel EV, Zangen A, Roth Y, Reti I, Braw Y, Levkovitz Y. H-coil repetitive transcranial magnetic stimulation for the treatment of bipolar depression: an add-on, safety and feasibility study. World J Biol Psychiatry 2011; 12(2): 119-26.

[13] Gershon A, Dannon P, Grunhaus L. Transcranial magnetic stimulation in the treatment of depression. Am J Psychiatry 2003; 160(5): 835-45.

[14] Hadley D, Anderson BS, Borckardt JJ, et al. Safety, tolerability, and effectiveness of high doses of adjunctive daily left prefrontal repetitive transcranial magnetic stimulation for treatment-resistant depression in a clinical setting. J ECT 2011, 27: 18-25.

[15] Sakkas P, Mihalopoulou P, Mourtzouhou P, et al. Induction of mania by rTMS: report of two cases. Eur Psychiatry 2003; 18: 1968.

[16] Available at: www.accessdata.fda.gov/cdrh_docs/pdf8/K083538.pdf - 2009-01-07.

[17] Mitchell P, Loo C. Transcranial magnetic stimulation for depression. Aust N Z J Psychiatry 2006; 40(5): 406-13.

[18] Rachid F, Bertschy G. Safety and efficacy of repetitive transcranial magnetic stimulation in the treatment of depression: a critical appraisal of the last 10 years. Neurophysiol Clin 2006; 36(3): 157-83.

[19] American Psychiatric Association. Diagnostic and statistical manual of mental disorders. 4th ed (DSM-IV). Washington, DC: APA 1994.

[20] World Health Organization: The ICD-10 Classification of Mental and Behavioural Disorders: Diagnostic Criteria for Research. Geneva: WHO 1993.

[21] Martin JL, Barbanoj MJ, Schlaepfer TE, et al. Transcranial magnetic stimulation for treating depression. Cochrane Database Syst Rev 2001; 2: CD003493.

[22] Hamilton M. A rating scale for depression. J Neurol Neurosurg Psychiatry 1960; 23: 56.

[23] Beck AT, Ward CH, Mendelson M, Mock J, Erbaugh J. An inventory for measuring depression. Arch Gen Psychiatry 1961; 4: 56171.

[24] McNamara B, Ray JL, Arthurs OJ, Boniface S. Transcranial magnetic stimulation for depression and other psychiatric disorders. Psychol Med 2001; 31(7): 1141-6.

[25] Holtzheimer PE 3rd, Russo J, Avery DH. A meta-analysis of repetitive transcranial magnetic stimulation in the treatment of depression. Psychopharmacol Bull 2001; 35(4): 149-69.

[26] Burt T, Lisanby SH, Sackeim HA. Neuropsychiatric applications of transcranial magnetic stimulation: a meta analysis. Int J Neuropsychopharmacol 2002; 5(1): 73-103.

[27] Kozel FA, George MS. Meta-analysis of left prefrontal repetitive transcranial magnetic stimulation (rTMS) to treat depression. J Psychiatr Pract 2002; 8(5):270-5.

[28] Speer AM, Kimbrell TA, Willis MW, et al. Baseline absolute blood flow measured with oxygen-15 PET predicts differential antidepressant response to $1 \mathrm{~Hz}$ versus $20 \mathrm{~Hz}$ rTMS. 152nd Annual Meeting of the American Psychiatric Association. Washington DC; 1999; Abstract NR191. 
[29] Martin JL, Barbanoj MJ, Schlaepfer TE, Thompson E, Pérez V, Kulisevsky J. Repetitive transcranial magnetic stimulation for the treatment of depression. Br J Psychiatry 2003; 182: 480-91.

[30] Klein E, Krenin I, Chistyakov A, et al. Therapeutic efficacy of right prefrontal slow repetitive transcranial stimulation in major depression. Arch Gen Psychiatry 1999; 56(4): 315-20.

[31] Berman RM, Narasimhan M, Sanacora G, et al. A randomized clinical trial of repetitive transcanial stimulaion in the treatment of major depression. Biol Psychiatry 2000; 47(4): 332-7.

[32] Manes F, Jorge R, Morcuende M, Yamada T, Paradiso S, Robinson RG. A controlled study of repetitive transcranial magnetic stimulation as a treatment of depression in the elderly. Int Psychogeriatr 2000; 13(2): 225-31.

[33] Hotopf M, Churchill R, Lewis G. Pragmatic randomised controlled trials in psychiatry. Br J Psychiatry 1999; 175: 217-23.

[34] Couturier JL. Efficacy of rapid-rate repetitive transcranial magnetic stimulation in the treatment of a depression: a systematic review and meta-analysis. J Psychiatry Neurosci 2005; 30(2): 83-90.

[35] Lisanby SH, Gutman D, Luber B, Schroeder C, Sackeim HA. Sham TMS: intracerebral measurement of the induced electrical field and the induction of motor-evoked potentials. Biol Psychiatry 2001; 49(5): 460-3.

[36] Hermann LL, Ebmeier KP. Factors modifying the efficacy of transcranial magnetic stimulation in the treatment of depression: A Review. J Clin Psychiatry 2006; 67(12):1870-6.

[37] Jorge RE, Robinson RG, Tateno A, et al. Repetitive transcranial magnetic stimulation as treatment of poststroke depression: a preliminary study. Biol Psychiatry 2004; 55(4): 398-405.

[38] Kauffmann CD, Cheema MA, Miller BE. Slow right prefrontal transcranial magnetic stimulation as a treatment for medicationresistant depression: a double-blind, placebo-controlled study. Depress Anxiety 2004; 19(1): 59-62.

[39] Gross M, Nakmura L, Pascual-Leone A, Fregni F. Has ripetitive transcranial magnetic stimulation ( $\mathrm{r}$ TMS) treatment for depression improbe? A systematic review ad meta-analysis comparing the recent vs. the earlier r TMS studies. Acta Psychiatr Scand 2007; 116(3): 165-73.

[40] Januel D, Dumortier G, Verdon CM, et al. A double-blind sham controlled study of right prefrontal repetitive transcranial magnetic stimulation (r-TMS): therapeutic and cognitive effect in medication free unipolar depression during 4 weeks. Prog Neuropsychopharmacol Biol Psychiatry 2006; 30(1): 126-30.

[41] Avery DH, Holtzheimer PE, Fawaz W, et al. A controlled study of repetitive transcranial magnetic stimulation in medication-resistant major depression. Biol Psychiatry 2006, 59: 187-94.

[42] Rossini D, Magri L, Lucca A, Giordani S, Smeraldi E, Zanardi R. Does r-TMS hasten the response to escitalopram, sertaline, or venlafaxine in patients with major depressive disorder? A doubleblind, randomized, sham-controlled trial. J Clin Psychiatry 2005; 66(12): 1569-75.

[43] Tamas RL, Menkes D, El-Mallakh RS. Stimulating research: a prospective, randomized, double-blind, sham-controled study of slow trascranial magnetic stimulation in depressed bipolar patients. J Neuropsychiatry Clin Neurosci 2007; 19(2): 198-9.

[44] Dell'Osso B, Altamura AC. Augmentative transcranial magnetic stimulation (TMS) combined with brain navigation in drugresistant rapid cycling bipolar depression: a case report of acute and maintenance efficacy. World J Biol Psychiatry 2009; 10(4 Pt 2): 673-6.

[45] Lam RW, Chan P, Wilkins-Ho M, Yatham LN. Repetitive transcranial magnetic stimulation for treatment resistant-depression: a systematic review and metaanalysis. Can J Psychiatry 2008; 53(9): 621-31.

[46] Fitzgerald PB, Huntsman S, Gunewardene R, Kulkarni J, Daskalakis ZJ. A randomized trial of low-frequency rightprefrontal-cortex transcranial magnetic stimulation as augmentation in treatment-resistant major depression. Int J Neuropsychopharmacol 2006; 9(6): 655-66.

[47] Turnier-Shea Y, Bruno R, Pridmore S. Daily and spaced treatment with transcranial magnetic stimulation in major depression: a pilot study. Aust N Z J Psychiatry 2006; 40(9): 759-63.

[48] Fitzgerald PB, Hoy K, McQueen S, et al. Priming stimulation enhances the effectiveness of low-frequency right prefrontal cortex transcranial magnetic stimulation in major depression. J Clin Psychopharmacol 2008; 28(1): 52-8.

[49] Loo C, Mitchell P, Sachdev P, Loo C, Mitchell P, Sachdev P. Double-blind controlled investigation of transcranial magnetic stimulation for the treatment of resistant major depression. Am J Psychiatry 1999; 156(6): 946-8.

[50] Bortolomasi M, Minelli A, Fuggetta G, et al. Long-lasting effects of high frequency repetitive transcranial magnetic stimulation in major depressed patients. Psychiatry Res 2007; 150(2): 181-6.

[51] Moser DJ, Jorge RE, Manes F, Paradiso S, Benjamin ML, Robinson RG. Improved executive functioning following repetitive transcranial magnetic stimulation. Neurology 2002; 58(8): 1288-90.

[52] Avery DH, Holtzheimer PE III, Fawaz W, et al. Transcranial magnetic stimulation reduces pain in patients with major depression: a sham-controlled study. J Nerv Ment Dis 2007; 195(5): 378-81.

[53] Pascual-Leone A, Rubio B, Pallardo F, Catalá MD. Rapid-rate transcranial magnetic stimulation of left dorsolateral prefrontal cortex in drug-resistant depression. Lancet 1996; 348(9022): 233-7.

[54] Miniussi C, Bonato C, Bignotti S, et al. Repetitive transcranial magnetic stimulation (rTMS) at high and low frequency: an efficacious therapy for major drug-resistant depression? Clin Neurophysiol 2005; 116(5): 1062-71.

[55] Garcia-Toro M, Mayol A, Arnillas H, et al. Modest adjunctive benefit with transcranial magnetic stimulation in medicationresistant depression. J Affect Disord 2001; 64(2-3): 271-5.

[56] Bretlau LG, Lunde M, Lindberg L, Undén M, Dissing S, Bech P. Repetitive transcranial magnetic stimulation (rTMS) in combination with escitalopram in patients with treatment-resistant major depression: a double-blind, randomised, sham-controlled trial. Pharmacopsychiatry 2008; 41(2): 41-7.

[57] Shutter DJ. Antidepressant efficacy of high-frequency transcranial magnetic stimulation over the left dorsolateral prefrontal cortex in double-blind sham-controlled designs: a meta-analysis. Psychol Med 2009; 39(1): 65-75.

[58] Shutter DJ. Quantitative review of the efficacy of slow-frequency magnetic brain stimulation in major depressive disorder. Psychol Med 2010; 40(11): 1789-95.

[59] Brunoni AR, Lopes M, Kaptchuk TJ, Fregni F. Placebo response of non-pharmacological and pharmacological trials in major depression: a systematic review and meta-analysis. PLoS One 2009; 4(3): e4824.

[60] Slotema CW, Blom JD, Hoek HW, Sommer IE. Should we expand the toolbox of psychiatric treatment methods to include Repetitive Transcranial Magnetic Stimulation (rTMS)? A meta-analysis of the efficacy of rTMS in psychiatric disorders. J Clin Psychiatry 2010; 71(7): 873-84.

[61] Machii K, Cohen D, Ramos-Estebanez C, Pascual-Leone A. Safety of r-TMS to non-motor cortical areas in healty partecipants and patients. Clin Neurophysiol 2006; 117(2): 455-71.

[62] Figiel GS, Epstein C, McDonald WM, et al. The use of rapid-rate transcranial magnetic stimulation (rTMS) in refractory depressed patients. J Neuropsychiatry Clin Neurosci 1998; 10(1): 20-5.

[63] Conca A, Konig P, Hausmann A. Trancsranial Magnetic stimulation induces "pseudoabsence seizure". Acta Psychiatr Scand 2000; 101(3): 246-8.

[64] Janicak PG, O'Reardon JP, Sampson SM, et al. Transcranial magnetic stimulation in the treatment of major depressive disorder: a comprehensive summary of safety experience from acute exposure, extended exposure, and during reintroduction treatment. J Clin Psychiatry 2008; 69(2): 1191-2.

[65] Loo CK, Mitchell PB. A review of the efficacy of transcranial magnetic stimulation (TMS) treatment for depression, and current and future strategies to optimize efficacy. J Affect Disord 2005; 88(3): 255-67.

[66] Daskalakis ZJ, Levinson AJ, Fitzgerald PB. Repetitive transcranial magnetic stimulation for major depressive disorder: a review. Can J Psychiatry 2008; 53(9): 621-31.

[67] O'Reardon JP, Solvason HB, Janicakc PG, et al. Efficacy and safety of transcranial magnetic stimulation in the acute treatment of major depression: A Multisite Randomized Controlled Trial. Biol Psychiatry 2007; 62(11): 1208-16.

[68] Schlaepfer TE, George MS, Mayberg H. WFSBP Guidelines on Brain Stimulation Treatments in Psychiatry. World J Biol Psychiatry $2010 ; 11(1): 2-18$. 
[69] Dell'osso B, D'Urso N, Castellano F, Ciabatti M, Altamura AC. Long-term efficacy after acute augmentative repetitive transcranial magnetic stimulation in bipolar depression: A 1-year follow-Up Study. J ECT. (in press).

[70] Janicak PG, Nahas Z, Lisanby SH, et al. Durability of clinical benefit with transcranial magnetic stimulation (TMS) in the treatment of pharmacoresistant major depression: assessment of relapse during a 6-month, multisite, open-label study. Brain Stimul 2010; 3(4): 187-99.

[71] Anderson BS, Kavanagh K, Borckardt JJ, et al. Decreasing procedural pain over time of left prefrontal rTMS for Depression: initial results from the Open-Label phase of multisite trial (OPT-TMS). Brain Stimul 2009; 2(2): 88-92.

Received: March 29, 2011

(C) Dell'Osso et al.; Licensee Bentham Open.

This is an open access article licensed under the terms of the Creative Commons Attribution Non-Commercial License (http://creativecommons.org/licenses/by-nc/3.0/) which permits unrestricted, non-commercial use, distribution and reproduction in any medium, provided the work is properly cited. 